\title{
Potential interest of InsR, CPT1A, SLC27A2, FASN and PPAR $\alpha$ expression in blood cells as biomarkers of dyslipidemia in children
}

J. Sánchez ${ }^{1}$, T. Priego ${ }^{1}$, C. Picó ${ }^{1}$, W. Ahrens $^{2}$, S. DeHenauw ${ }^{3}$, A. Fraterman ${ }^{4}$, L. Iacoviello ${ }^{5}$, S. Mårild $^{6}$, D. Molnár ${ }^{7}$, L. A. Moreno ${ }^{8}$, J. Peplies ${ }^{2}$, P. Russo ${ }^{9}$, A. Siani ${ }^{9}$, M. Tornaritis ${ }^{10}$, T. Veidebaum ${ }^{11}$ and A. Palou ${ }^{1}$ on behalf of the Idefics Consortium

${ }^{1}$ Laboratory of Molecular Biology, Nutrition and Biotechnology (Nutrigenomics), University of the Balearic Islands (UIB) and CIBER Fisiopatología de la Obesidad y Nutrición (CIBEROBN), Palma de Mallorca, Spain, ${ }^{2}$ Bremen Institute for Prevention Research and Social Medicine, University of Bremen, Bremen, Germany, ${ }^{3}$ Department of Public Health/ Department of Movement and Sport Sciences, Faculty of Medicine and Health Sciences, Ghent University, Ghent, Belgium, ${ }^{4}$ Laboratoriumsmedizin Dortmund, Eberhard \& Partner, Dortmund, Germany, ${ }^{5}$ Centre for High Technology Research and Education in Biomedical Sciences, Universita Cattolica del Sacro Cuore, Campobasso, Italy, ${ }^{6}$ Queen Silvia Childrens Hospital, Department of Pediatrics, Göteburg University, Gothenburg, Sweden, ${ }^{7}$ Department of Pediatrics, University of Pécs, Pécs, Hungary, ${ }^{8}$ GENUD (Growth, Exercise, Nutrition and Development) research group, University of Zaragoza, Zaragoza, Spain, ${ }^{9}$ Institute of Food Sciences, Unit of Epidemiology and Population Genetics, National Research Council, Avellino, Italy, ${ }^{10}$ Research and Education of Child Health Institute, Strovolos, Cyprus and ${ }^{11}$ National Institute for Health Development, Tervise Arengu Instituut, Tallinn, Estonia

The alarming increase in obesity worldwide is of concern mainly because of the associations of obesity with the metabolic syndrome, characterized by central obesity, insulin resistance, hyperglycemia, dyslipidemia and hypertension; all risk factors for cardiovascular diseases and type 2 diabetes mellitus. Therefore, the identification of new early biomarkers of susceptibility is relevant to intervene with lifestyle and nutritional changes before the disease is manifested. Blood cells are useful as potential source of transcript-based biomarkers in human samples because they are easily accessible and changes in gene expression in these cells may reflect processes occurring at internal organs. Thus, the objective of this study was to evaluate, in blood samples of normal weight and overweight children, whether the expression levels of selected genes involved in energy homeostasis, such as InsR, CPT1A, SLC27A2, FASN and PPAR $\alpha$, are related with the dyslipidemic state associated with obesity. Blood samples were obtained from 306 normal-weight and overweight children, aged 2-9 years, from 8 different European countries. Whole-blood mRNA levels were assessed by RT-qPCR. Overweight children displayed higher triglyceride levels than normal-weight children. However, overweight children with high expression levels of InsR or CPT1A, did not show the increase in triglyceride levels associated with fat accumulation, while no effect was found apparently in normal weight children. With respect to SLC27, FASN and PPAR $\alpha$, both normal weight and overweight children with high expression levels of these genes exhibited lower triglyceride levels. In conclusion, expression levels of InsR, CPT1A, SLC27, FASN or PPAR $\alpha$ in blood cells may be used as biomarkers of the metabolic status in overweight children, particularly in terms of triglyceridemia, potentially providing an early warning of future disorders.

This work was done as part of the IDEFICS Study (www.idefics.eu). This work has been supported by the European Community within the Sixth RTD Framework Programme, Contract No. 016181 (FOOD), the Spanish Government (grant AGL2006-27837-E to C.P.), and the Instituto de Salud Carlos III, Centro de Investigación Biomédica en Red Fisiopatología de la Obesidad y Nutrición, CIBERobn. The Laboratory is a member of the European Research Network of Excellence NuGO (The European Nutrigenomics Organization, EU Contract: n. FP6-506360). 